\title{
A Rapid Microtiter Assay to Evaluate Fungicide Sensitivity to Colletotrichum falcatum Isolates
}

\author{
Renato de Carvalho Menezes*, Mariana Guimarães Silva, Thayssa Monize Rosa Oliveira, Geisiane Alves Rocha, \\ Vanessa Duarte Dias, Renato Carrer Filho and Marcos Gomes da Cunha \\ Universidade Federal de Goiás, Goias, Brazil \\ *For correspondence: renato_cmenezes@hotmail.com \\ Received 05 August 2021; Accepted 16 December 2021; Published 30 January 2022
}

\begin{abstract}
Chemical control of sugarcane red rot, caused by Colletotrichum falcatum, forms part of integrated management of the disease. A rapid microtiter bioassay based on the colorimetric changes of resazurin dye was developed to evaluate the sensitivity of $C$. falcatum to the main chemical fungicide groups, including strobilurin, triazole, benzimidazole, isophthalonitrile and dithiocarbamate. There was no significant difference among the isolates in terms of growth inhibition for any of the active ingredients tested $(\alpha=0.01)$. The $C$. falcatum isolates showed almost similar sensitivity to various fungicides. The active ingredients varied in relation to fungitoxicity. Doses that inhibited $50 \%$ of $C$. falcatum growth were calculated as a percentage of resazurin reduction due to various fungicides. The colorimetric method used to assess the fungitoxicity of active ingredients to $C$. falcatum, combined with resazurin, proved a fast practical and efficient method. (C) 2022 Friends Science Publishers
\end{abstract}

Keywords: Chemical control; Fungitoxicity; Red rot; Resazurin; Sugarcane

\section{Introduction}

The fungus $C$. falcatum is the causal agent of sugarcane red rot, one of the most destructive diseases that affects the crop (Khan et al. 2011; Bharti et al. 2012; Sharma and Tamta 2015). Widely disseminated across all continents, sucrose yield losses of 50-70\% have been reported in infected stems (Santiago and Rossetto 2008). Earliest red rot epidemics were atributed to attach of Diatrea saccaralis, but the fungus was no longer $100 \%$ associated with Diatrea saccharalis, more aggressive variants do not need the hole of the insect to penetrate the stalk. With the absence of burned, the pathogen survives in the soil and crop residues after harvest and with each harvest the incidence and severity increases (Viswanathan 2010), it has become a very harmful disease to sugarcane crops in countries like Brazil and India (Viswanathan et al. 2020a).

The fungal pathogen exhibits enormous variation under fields conditions and the pathogenics variants emerge regularly in tune with deployment of new host varieties for cultivation making the resistant to susceptible referred as "varietal breakdown" (Viswanathan et al. 2020b). So, already many sugarcane varieties were replaced due to their breakdown to a new pathogenic strain, so chemical control may be more one possibility in the management of red rot in sugarcane crop. Given the losses caused by the disease, existence of pathogenic variation in C. falcatum and the emergence of new virulent pathotypes were documented over the decades (Viswanathan 2010; Viswanathan et al. 2020b).

Among the management strategies used, fungicides are an important supplementary tool in controlling different diseases (Nene and Thapliyal 1993). However, in the case of sugarcane red rot, information on the sensitivity of various $C$. falcatum isolates to different fungicides remains scarce, largely due to the lack of a practical and efficient method to conduct this assessment. Conventional techniques for evaluating the fungitoxicity of active ingredients in a fungicide-enriched growth medium by inhibiting mycelial growth and/or conidial germination are costly, laborious, time-consuming, require a significant amount of laboratory space to accommodate a large number of plates, cannot identify intermediate sensitivity to fungicides and preclude automated data collection (Rampersad and Teelucksingh 2012; Promega Corporation 2019).

An alternative strategy for assessing the fungitoxicity of active ingredients is resazurin, a stable, nontoxic watersoluble dye used as an indicator in oxidation-reduction reactions. Resazurin has been used to measure cell proliferation, as well as the viability and cytotoxicity of different cell types in medical research (Invitrogen Molecular Probes 2021; ThermoFisher Scientific 2021). As such, the present study aimed to (i) adjust a colorimetric method for assessing the sensitivity of $C$. falcatum isolates

To cite this paper: Menezes RDC, MG Silva, TMR Oliveira, GA Rocha, VD Dias, RC Filho, MGD Cunha (2022). A rapid microtiter assay to evaluate fungicide sensitivity to Colletotrichum falcatum isolates. Intl J Agric Biol 27:83-88 
to the main fungicide chemical groups strobilurin, triazole, benzimidazole, isophthalonitrile, and dithiocarbamate and (ii) evaluate the fungitoxicity and sensitivity of the different C. falcatum isolates of the active ingredients tested.

\section{Materials and Methods}

\section{Preparation of the conidial suspension}

Eighteen fungal isolates were obtained from monoconidial C. falcatum cultures belonging to the microorganism collection of the Plant Pathology Laboratory at the School of Agronomy of the Federal University of Goias, collected in the state of Goias in the 2017 growing season. For the production of conidia, the isolates were cultivated in Petri dishes containing potato dextrose agar (PDA) medium for 20 days at $28-30^{\circ} \mathrm{C}$, under constant darkness.

In order to determine the optimal conidia concentration to test the sensitivity to fungicides of $18 \mathrm{C}$. falcatum isolates, suspensions were prepared at initial concentrations of $10^{4}$, $10^{5}$ and $10^{6}$ conidia $\mathrm{mL}^{-1}$ for one C. falcatum isolate (CFF 12). The conidial suspension $(60 \mu \mathrm{L}), 140 \mu \mathrm{L}$ of Czapek Dox Broth medium $+0.05 \%$ agar and $10 \mu \mathrm{L}$ of $0.02 \%$ resazurin were deposited into a clear flat bottom polystyrene microplate. Each conidial suspension concentration was deposited in three repetitions (microplate wells). The negative control used was $60 \mu \mathrm{L}$ sterile water without conidia, also in three repetitions. The microplate was incubated in a biochemical oxygen demand (BOD) incubator at $28-30^{\circ} \mathrm{C}$, under constant darkness. In order to determine the ideal incubation period, the resazurin reduction percentage was evaluated after 48 and $72 \mathrm{~h}$ of incubation.

\section{Fungicide sensitivity testing}

The following fungicides were tested: Amistar ${ }^{\circledR}$ WG $500 \mathrm{~g}$ $\mathrm{kg}^{-1}$ of Azoxystrobin, obtained from Syngenta), Tilt ${ }^{\circledR}(250$ $\mathrm{g} \mathrm{L}^{-1}$ of propiconazole, Syngenta), Carbendazim (97\%, Sigma-Aldrich), Manzate 800 (800 $\mathrm{g} \mathrm{kg}^{-1}$ of mancozeb, UPL), Bravonil ${ }^{\circledR} 720$ (720 g L-1 of chlorothalonil, Syngenta) and Cercobin 700 WP (700 g kg$~^{-1}$ of thiophanate-methyl, Iharabras). Each fungicide was precisely weighed and dissolved in Czapek Dox Broth medium $+0.05 \%$ agar (used as a dispersing agent) in serial dilutions. The active ingredient concentrations used were $0 ; 0.01 ; 0.1 ; 0.5 ; 1 ; 5$; 10 and $50 \mu \mathrm{g} \mathrm{mL}^{-1}$.

Suspensions with a concentration $10^{5}$ conidia $\mathrm{mL}^{-1}$ were prepared for each of the 18 isolates. Each well received $60 \mu \mathrm{L}$ of conidial suspension, $140 \mu \mathrm{L}$ of Czapek Dox Broth medium $+0.05 \%$ agar, active ingredients at the doses previously mentioned and $10 \mu \mathrm{L}$ of $0.02 \%$ resazurin. For strobilurin, $10 \mu \mathrm{L}$ of salicylhydroxamic acid (SHAM) was added to the well to suppress the alternative oxidase pathway (Ma et al. 2006). Active ingredient doses were tested in three wells for each isolate. The microplates were sealed and incubated in a BOD incubator at $28-30^{\circ} \mathrm{C}$ under constant darkness. The resazurin reduction percentage was evaluated after $48 \mathrm{~h}$ of incubation.

Absorbance of the microplate wells was read in an Epoch microplate spectrophotometer (Biotek, Vermont, United States) after $48 \mathrm{~h}$ of incubation, at wavelengths of $570 \mathrm{~nm}$ and $600 \mathrm{~nm}$, with the aid of GENE 5 software, version 2.0. The resazurin reduction percentage was estimated using the formula provided by the manufacturer of alamarBlue ${ }^{\circledR}$ reagent (Cox et al. 2009).

\section{Data analysis}

Data were analyzed in $\mathrm{R}$ software version 3.6.2. For each dose of active ingredient (dose y), the growth inhibition percentage (GIP) of the isolate was calculated in relation to dose zero (dose 0) using the estimated resazurin reduction percentage, which indirectly measures initial $C$. falcatum growth. For each active ingredient, the GIP as a function of dose and isolates was submitted to analysis of variance, using the ExpDes package of $\mathrm{R}$ software. The nls and nlstools packages were used for nonlinear regression adjustment based on the model proposed on MichaelisMenten model, where GIP is a function of the active ingredient dose.

\section{Results}

\section{Adjustment of spore concentration and incubation time}

Preliminary tests to adjust the spore concentration and incubation time were assessed by analysis of variance and Tukey's test $(\alpha=0.01)$. A concentration of $10^{5}$ conidia $\mathrm{mL}^{-1}$ provided the greatest resazurin reduction, with no significant difference between incubation times of 48 and $72 \mathrm{~h}$, confirming the stability of the dye after $48 \mathrm{~h}$ of incubation.

\section{Sensitivity of $C$. falcatum isolates to fungicides}

For each active ingredient, the $C$. falcatum GIP as a function of dose and isolates was submitted to analysis of variance, with no significant difference in GIP between $C$. falcatum isolates $(\alpha=0.01)$ (data not shown). The regression curve that expresses the $C$. falcatum GIP as a function of dose, based on the model proposed by (Michaelis and Menten 1913), was adjusted for each active ingredient. Thus, all the regression curves were significant at $0.1 \%$ according to the F-test. The regression equations, coefficients of determination $\left(\mathrm{R}^{2}\right)$ and doses that inhibited $50 \%$ of growth $\left(\mathrm{EC}_{50}\right)$ for each active ingredient are described in Table 1.

The effects of the active ingredients varied in relation to fungitoxicity to $C$. falcatum. The sensitivity of $C$. falcatum to the molecules tested can be ranked in descending order based on $\mathrm{EC}_{50}$, as follows: azoxystrobin, propiconazole, carbendazim, mancozeb, chlorothalonil and thiophanate-methyl. Comparison of the fungitoxicity of the active ingredients is shown in Fig. 1 and 2. 


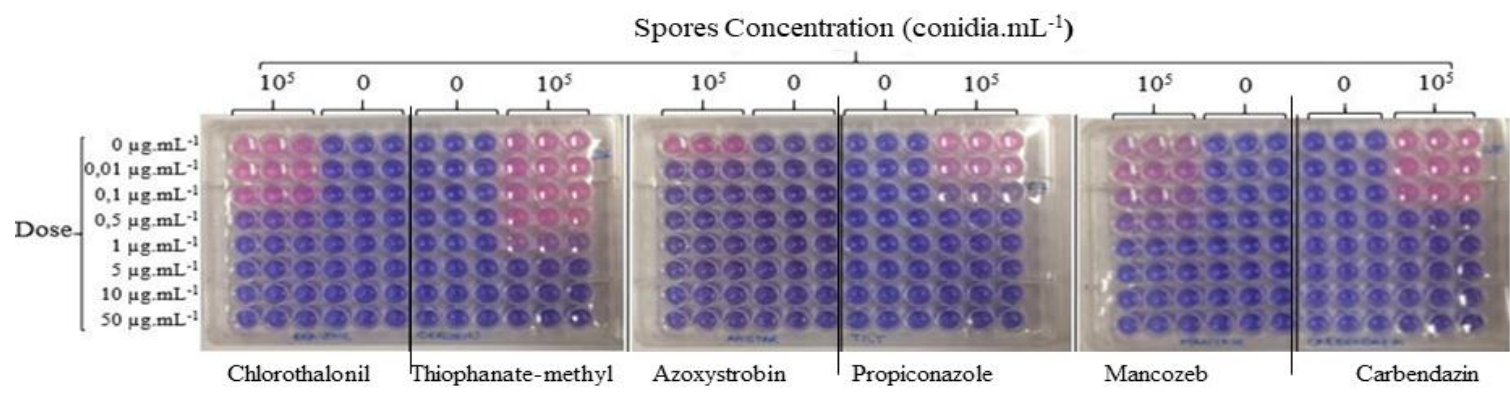

Fig. 1: Visualization of Colletotrichum falcatum growth inhibition by resazurin reduction as a function of fungicide doses.

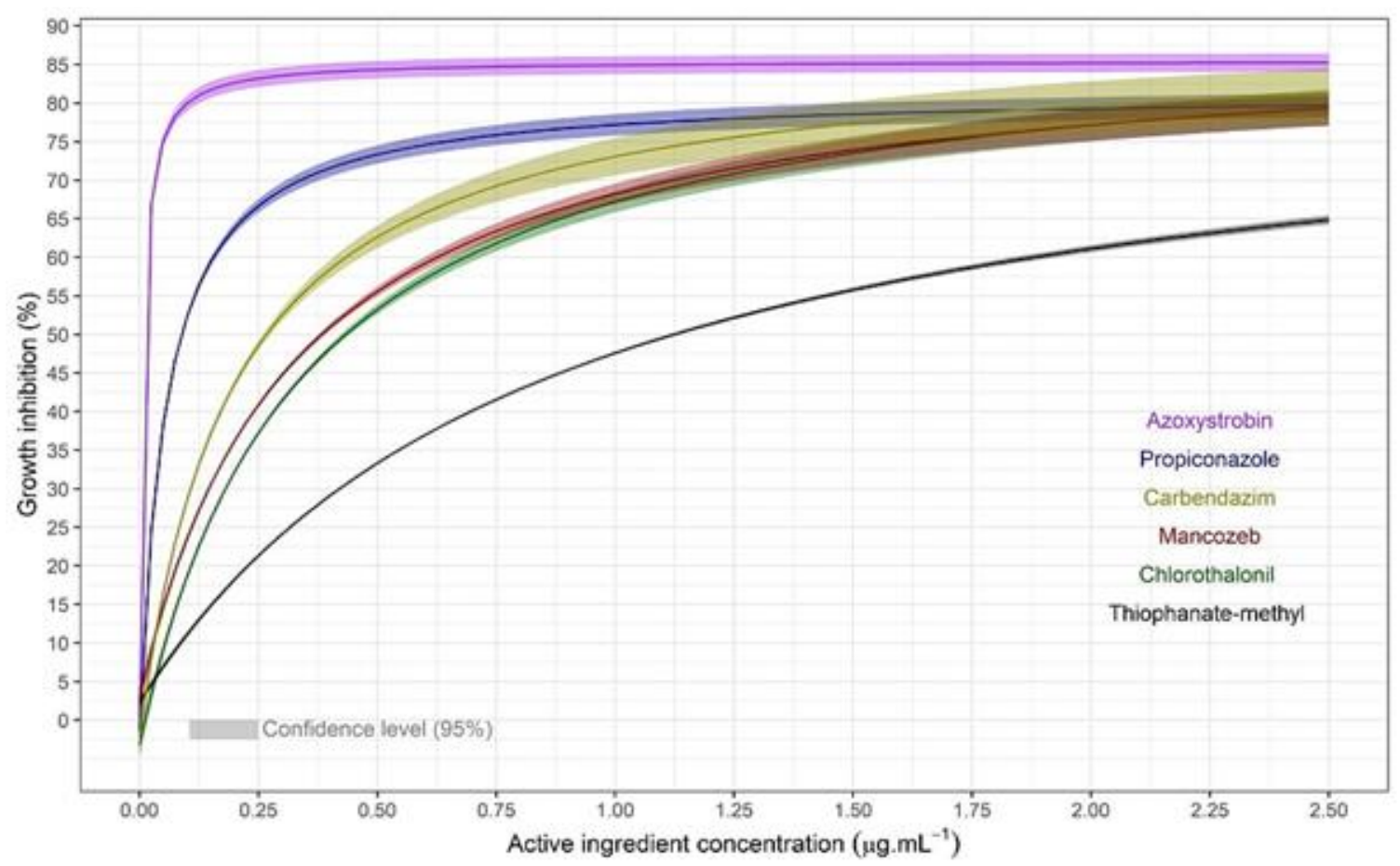

Fig. 2: Comparative regression curves of Colletotrichum falcatum growth inhibition as a function of fungicide fungitoxicity. CI: confidence interval

Table 1: Regression equation, coefficients $\mathrm{R}^{2}$ e $\mathrm{EC}_{50}$

\begin{tabular}{llll}
\hline Active Ingredient & Equation & $\mathrm{R}^{2}$ & $\mathrm{EC}_{50}\left(\mu \mathrm{g} \cdot \mathrm{mL}^{-1}\right)$ \\
\hline Azoxystrobin & $\mathrm{GIP}=-0.13+85.63$. Dose $/(0.0069+$ Dose $)$ & 0.994 & 0.0097 \\
Propiconazole & GIP $=-1.41+82.87$. Dose $/(0.0542+$ Dose $)$ & 0.989 & 0.0885 \\
Carbendazim & GIP $=-2.26+89.78$. Dose $/(0.1916+$ Dose $)$ & 0.931 & 0.2668 \\
Mancozebe & GIP $=2.84+86.09$. Dose $/(0.3161+$ Dose $)$ & 0.954 & 0.3829 \\
Chlorothalonil & GIP $=-3.24+93.56$. Dose $/(0.3281+$ Dose $)$ & 0.956 & 0.4332 \\
Thiofanate methil & GIP $=2.28+83.92$. Dose $/(0.8519+$ Dose $)$ & 0.987 & 1.1230 \\
\hline
\end{tabular}

\section{Discussion}

With respect to the spore concentration that optimizes resazurin reduction, the present study demonstrated that $10^{5}$ conidia $\mathrm{mL}^{-1}$ provided the best results after $48 \mathrm{~h}$ of incubation. Resazurin sensitivity to spore density is dependent on the fungal species studied and should be determined empirically for studies involving dye reduction in response to the fungitoxicity of pesticides to phytopathogenic fungi (Cox et al. 2009). Larson et al. (1997) analyzed the viability of corneal endothelial cells and Pelloux-Prayer et al. (1998) the viability of Botrytis cinereal conidia, with both studies reporting that densities lower than $10^{4}$ cells $\mathrm{mL}^{-1}$ required longer incubation times to reduce resazurin, whereas more than $10^{5}$ cells $\mathrm{mL}^{-1}$ produced inconsistent results. Larson et al. (1997) also found that high 
cell densities and prolonged incubation times cause resazurin to reach an undesirable plateau because the (blue) dye is reduced to resorufin (pink which, in turn, is reduced to hydroresorufin (colorless). The reduction percentage for resorufin is higher than that of hydroresorufin (Rampersad 2011).

In regard to the incubation times tested, resazurin reduction stabilized between 48 and $72 \mathrm{~h}$. This indicates that the dye reached its reduction limit at $48 \mathrm{~h}$, which was the time established for testing to assess $C$. falcatum sensitivity to fungicides. Prolonged incubation times compromise resazurin reduction, as reported by Larson et al. (1997).

There was no significant difference in growth inhibition, estimated by the resazurin reduction percentage $(\alpha=0.01)$, between isolates for any of the active ingredients tested. As such, the $C$. falcatum isolates studied showed equal sensitivity to azoxystrobin, propiconazole, carbendazim, mancozeb, chlorothalonil and thiophanate-methyl. Ghazanfar et al. (2017) reported that Tilt ${ }^{\circledR}$ (250 g.L $\mathrm{L}^{-1}$ of propiconazole) was more efficient than mancozebe at inhibiting the mycelial growth of the fungus and Nenhuma fonte bibliográfica especificada. It was found more effective at controlling sugarcane red rot.

In the present study, the active ingredient propiconazole was the second most toxic to the pathogen (Fig. 1 and 2). In research on other chemical molecules, Nikhil and Sahu (2014) observed that carbendazim was more effective than propiconazole at inhibiting mycelial growth, whereas Abbas et al. (2016) reported that mancozebe was most effective, followed by propiconazole, and found that benomyl, tebuconazole and mancozeb were more efficient than propiconazole. Although benomyl and tebuconazole were not tested here, carbendazim was only less toxic to $C$. falcatum than azoxystrobin and propiconazole, with mancozeb ranked fourth in terms of fungitoxicity to the molecules tested (Fig. 1 and 2).

Studies that evaluate the sensitivity of $C$. falcatum to different fungicides measure the effect of different products on the mycelial growth of the fungus and/or conidial germination in a culture medium, generally with doses above $5 \mu \mathrm{g} \mathrm{mL}^{-1}$. However, a number of investigations do not indicate whether the dose refers to active ingredients or commercial products (Bharti et al. 2014; Ghazanfar et al. 2017). In our study, doses greater than $5 \mu \mathrm{g} \mathrm{mL}^{-1}$ were too high, which precluded determining the fungitoxicity of azoxystrobin, propiconazole, carbendazim, mancozeb, chlorothalonil, and thiophanate-methyl (Fig. 1). Although the aforementioned studies evaluated the sensitivity of the pathogen to different fungicides, none provided data on the active ingredient dose $\left(\mathrm{EC}_{50}\right)$ that inhibited $50 \%$ of mycelial growth and/or $50 \%$ of conidial germination, which precluded comparison with the $\mathrm{EC}_{50}$ values found here (Nikhil and Sahu 2014; Bharti et al. 2014; Abbas et al. 2016).

$\mathrm{EC}_{50}$ has been estimated for other species of the genus Colletotrichum. Analysis of the conidial germination of $C$. gloeosporioides and $C$. capsica isolates showed $\mathrm{EC}_{50}$ values from 0.009 to $0.091 \mu \mathrm{g} \mathrm{mL}^{-1}$ for both species (Li et al. 2005). In our study, the $\mathrm{EC}_{50}$ of azoxystrobin for $C$. falcatum, calculated based on the estimated resazurin reduction percentage, was $0.0097 \mu \mathrm{g} \mathrm{mL}^{-1}$, within the range described above (Table 1). The $\mathrm{EC}_{50}$ values for the active ingredients propiconazole and thiophanate-methyl were estimated by the mycelial growth of $C$. cereale isolates and varied from 0.025 to $0.35 \mu \mathrm{g} \mathrm{mL}^{-1}$ and 0.14 to $2.3 \mu \mathrm{g} \mathrm{mL}^{-1}$, respectively (Wong and Midland 2007; Wong et al. 2008). In the present study, the $\mathrm{EC}_{50}$ values of propiconazole and thiophanate-methyl for $C$. falcatum were $0.0885 \mu \mathrm{g} \mathrm{mL}^{-1}$ and $1.123 \mu \mathrm{g} \mathrm{mL}^{-1}$, respectively, similar to those reported for $C$. cereale.

The $\mathrm{EC}_{50}$ values of carbendazim and mancozeb for $C$. acutatum isolates estimated, respectively, by the inhibition of mycelial growth and conidial germination, were 0.1946 $\mu \mathrm{g} \mathrm{mL}^{-1}$ for carbendazim and between 0.24 and $0.85 \mu \mathrm{g} \mathrm{mL}^{-1}$ for mancozeb (Cai et al. 2008; Gao et al. 2017). For $C$. falcatum, our study demonstrated $\mathrm{EC}_{50}$ values of $0.2668 \mu \mathrm{g}$ $\mathrm{mL}^{-1}$ for carbendazim and $0.3829 \mu \mathrm{g} \mathrm{mL}^{-1}$ for mancozeb, calculated based on the estimated resazurin reduction percentage, similar to the values reported for C. acutatum.

The $\mathrm{EC}_{50}$ of chlorothalonil was estimated for $C$. truncatum and $C$. gloeosporioides by mycelial growth inhibition, with values from 0.23 to $1.14 \mu \mathrm{g} \mathrm{mL}^{-1}$ and 0.01 to $0.95 \mu \mathrm{g} \mathrm{mL}^{-1}$, respectively (Rampersad and Teelucksingh 2012). For $C$. falcatum, our study recorded an $\mathrm{EC}_{50}$ of 0.4332 $\mu \mathrm{g} \mathrm{mL} \mathrm{mL}^{-1}$ for chlorothalonil, calculated via the estimated resazurin reduction percentage, which is within the ranges reported for $C$. truncatum and $C$. gloeosporioides.

The aforementioned studies found similar $\mathrm{EC}_{50}$ values for the active ingredients in question for species from the genus Colletotrichum to those recorded to $C$. falcatum. Some of the studies calculated the $\mathrm{EC}_{50}$ for each isolate used and established a range for that dose. However, when isolates exhibit similar sensitivity to fungicides, as observed for the C. falcatum isolates tested here, $\mathrm{EC}_{50}$ is estimated for a set of fungal isolates. Application of the fungicides showed a reduction in red rot incidence over control but the extent of reduction varied considerably (10.6-39.4\% reduction) across the treatments over the 2 years. Overall, among the seven fungicides evaluated, sett treatment with two fungicides (Tebuconazole + Trifloxystrobin and Thiophanate methyl) consistently resulted in $>30 \%$ reduction in red rot incidence over control in both years along with significantly higher NMC over control (Joshi 2021).

Resazurin has been used successfully in active ingredient fungitoxicity tests in several agronomic studies. Research has reported a high correlation between the results obtained with the traditional method and the resazurin-based assay (Cox et al. 2009; Vega et al. 2012). In summary, we found that the resazurin method is a faster, low-cost alternative to mycelial growth assays for $C$. falcatum. This assay may be a more reliable indicator of fungicide resistance because it uses spores instead of mycelia ( $\mathrm{Hu}$ et al. 2007). Furthermore, it can provide sugarcane growers 
with needed information about the sensitivity of isolates collected from their orchards in a more cost-effective manner. The technology also adds simplicity to the detection of resistance, especially when quantification is not desired, and results can be taken visually.

\section{Conclusion}

The colorimetric method used to assess the fungitoxicity of active ingredients to $C$. falcatum, combined with resazurin, is fast, practical, efficient and viable for application in large sets of isolates. All the $C$. falcatum isolates studied exhibited equal sensitivity to azoxystrobin, propiconazole, carbendazim, mancozeb, chlorothalonil and thiophanatemethyl. The active ingredients displayed different levels of toxicity to $C$. falcatum, ranked in descending order as follows: azoxystrobin, propiconazole, carbendazim, mancozeb, chlorothalonil and thiophanate-methyl.

\section{Acknowledgements}

The authors are grateful to the EMBRAPA for providing necessary facilities to use the microplate spectrophotometer at the institution. We would like to thank $\mathrm{CNPq}$ and Capes for financial support. This work would not have been possible without equipment provides for FAPEG.

\section{Author Contributions}

RCM and MGC planned the experiments, RCM, MGS and TMRO executed the tests, GAR, RCM, VDD and RCF made the write up and statistically analyzed the data.

\section{Conflict of Interest}

All authors declare no conflict of interest

\section{Data Availability}

Data presented in this study will be available on a fair request to the corresponding author.

\section{Ethics Approval}

Not applicable in this paper.

\section{References}

Abbas H, SA Anwar, N Javed, MA Iqbal, N Abid (2016). Morphological variability among isolates of Colletotrichum falcatum Went; infecting four cultivars of sugarcane. Pak J Phytopathol 2:101-104

Bharti Y, A Kumar, D Sharma, S Singh, D Shukla (2014). Morphological, physiological and pathological variations among isolates of Colletotrichum falcatum that cause red rot of sugarcane. Afr $J$ Microbiol Res 8:1040-1049

Bharti Y, S Vishwakarma, A Kumar, A Singh, M Sharma, D Shukla (2012). Physiological and pathological aspects of some new isolates of Colletotrichum falcatum causing red rot disease in Saccharum spp. complex. Acta Phytopathol Entomol Hung 47:35-50
Cai ZY, JZ Li, JQ Wang, CX Zhang (2008). Sensitivity test of Colletotrichum gloeosporioides and Colletotrichum acutatum isolated from rubber to the fungicides. J Yunnan Agric Univ 23:787-790

Cox KD, K Quello, RJ Deford, JL Beckerman (2009). A rapid method to quantify fungicide sensitivity in the brown rot pathogen Monilinia fructicola. Plant Dis 93:328-331

Gao YY, LF He, BX Li, W Mu, J Lin, F Liu (2017). Sensitivity of Colletotrichum acutatum to six fungicides and reduction in incidence and severity of chili anthracnose using pyraclostrobin. Aust Plant Pathol 46:521-528

Ghazanfar MU, W Raza, SK Gonda (2017). Screening of sugarcane cultivars against colletotrichum falcatum causing red rot disease and its control with different fungicides under laboratory conditions. Pak J Phytopathol 29:103-110

Hu J, C Hong, EL Stromberg, GW Moormam (2007). Effects of propamocarb hydrochloride on mycelial growth, sporulation, and Infection by Phytophthora nicotianae isolates from Virginia nurseries. Plant Dis 91:414-420

Invitrogen Molecular Probes (2021). Available at: <https://www.thermofisher.com/br/en/home/brands/molecularprobes>. (Accessed: 11 May 2021)

Joshi D (2021). Exploring potential of different fungicides for management of red rot of sugarcane under sub-tropical India. Ind Phytopathol 74:1-7

Khan A, M Awais, W Raza, A Zia (2011). Identification of sugarcane lines with resistance to red rot. Pak J Phytopathol 23:98-102

Larson EM, DJ Doughman, DS Gregerson, WF Obritsch (1997). A new, simple, nonradioactive, nontoxic in vitro assay to monitor corneal endothelial cell viability. Invest Ophthal Vis Sci 38:1929-1933

Li HX, ZY Liu, JX Wang, MG Zhou (2005). Baseline sensitivity of Colletotrichum gloeosporioides and C. capsici from Capsium to azoxystrobin. Acta Phytopathol Sin 35:73-77

Ma Z, TJ Proffer, JL Jacobs, GW Sundin (2006). Overexpression of the $14 \alpha$-demethylase target gene (CYP51) mediates fungicide resistance in Blumeriella jaapii. Appl Environ Microbiol 72:2581-2585

Michaelis L, ML Menten (1913). Die Kinetik der Invertinwirkung. Biochem Z 49:333-369

Nene YL, PN Thapliyal (1993). Fungicides in plant disease control. International Science Publisher, New Delhi, India

Nikhil B, RK Sahu (2014). Evaluation of some fungicides, botanicals and essential oils against the fungus Colletotrichum falcatum causing red rot of sugarcane. Bioscan 9:175-178

Pelloux-Prayer A, B Priem, J Joseleau (1998). Kinetic evaluation of conidial germination of Botrytis cinerea by a spectrofluorometric method. Mycol Res 102:320-322

Promega Corporation. Protocols and Applications Guide. (2019). Available at: <https://www.promega.com.br/cnotes/cn016_18.htm>. (Accessed: 11 May 2021)

Rampersad SN (2011). A rapid colorimetric microtiter bioassay to evaluate fungicide sensitivity among Verticillium dahliae isolates. Plant Dis 95:248-255

Rampersad SN, LD Teelucksingh (2012). Differential responses of Colletotrichum gloeosporioides and $C$. truncatum isolates from different hosts to multiple fungicides based on two assays. Plant Dis 96:1526-1536

Santiago AD, R Rossetto (2008). Arvore do conhecimento: Cana-deaçúcar. AGEITEC-Agência Embrapa de Informação Tecnológica. Brasilia, Brazil

Sharma R, S Tamta (2015). A review on red rot: The cancer of sugarcane. $J$ Plant Pathol Microbiol 6:1-7

ThermoFisher Scientific (2021). Available at: <https://www.thermofisher.com/order/catalog/product/Y00-025>. (Accessed: 11 October2021)

Vega B, D Liberti, PF Harmon, MM Dewdney (2012). A rapid resazurinbased microtiter assay to evaluate QoI sensitivity for Alternaria alternata isolates and their molecular characterization. Plant Dis 96:1262-1270

Viswanathan R (2010). Plant disease: Red rot of sugarcane. Anmol Publications Pvt Ltd. New Delhi, India 
Menezes et al. / Intl J Agric Biol, Vol 27, No 1, 2022

Viswanathan R, R Selvakumar, K Manivannan, R Nithyanandam, K Kaverinathan (2020a). Pathogenic behaviour of soil borne inoculum of Colletotrichum falcatum in causing red rot in sugarcane varieties with varying disease resistance. Sugar Technol 22:485-497

Viswanathan R, P Padmanaban, R Selvakumar (2020b). Emergence of new pathogenic variants in Colletotrichum falcatum, stalk infecting ascomycete in sugarcane: Role of host varieties. Sugar Technol $22: 473-484$
Wong FP, SL Midland (2007). Sensitivity distributions of California populations of Colletotrichum cereale to the DMI fungicides propiconazole, myclobutanil, tebuconazole, and triadimefon. Plant Dis $91: 1547-1555$

Wong FP, KADL Cerda, R Hernandez-Martinez, SL Midland (2008). Detection and characterization of benzimidazole resistance in California populations of Colletotrichum cereale. Plant Dis 92:239-246 